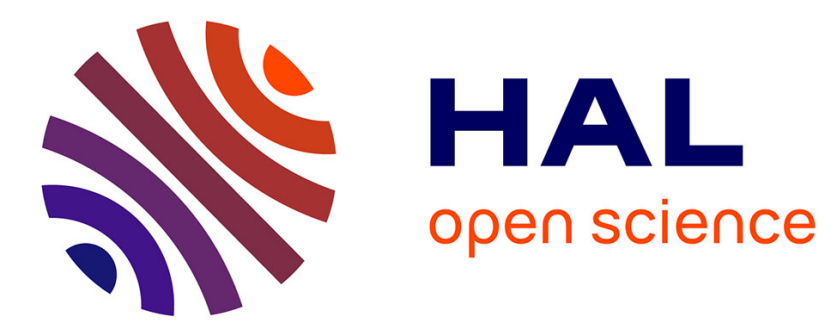

\title{
Les retours compulsifs des artistes contemporains aux images ready-made
}

\author{
Danièle Méaux
}

\section{To cite this version:}

Danièle Méaux. Les retours compulsifs des artistes contemporains aux images ready-made. L'esprit créateur, 2021, Rhétoriques du document, 61 (2). hal-03366915

\section{HAL Id: hal-03366915 https://hal.science/hal-03366915}

Submitted on 6 Oct 2021

HAL is a multi-disciplinary open access archive for the deposit and dissemination of scientific research documents, whether they are published or not. The documents may come from teaching and research institutions in France or abroad, or from public or private research centers.
L'archive ouverte pluridisciplinaire HAL, est destinée au dépôt et à la diffusion de documents scientifiques de niveau recherche, publiés ou non, émanant des établissements d'enseignement et de recherche français ou étrangers, des laboratoires publics ou privés. 


\section{Les retours compulsifs aux images ready made}

\section{Danièle Méaux}

Depuis les années 80, des artistes (ou des photographes) français s'approprient de façon répétée, voire obsessionnelle des documents visuels préexistants. Le recours croissant à des représentations « déjà-là » a souvent été corrélé au déferlement actuel des images, susceptible d'incliner certains à ne plus vouloir créer de nouveaux objets ${ }^{1}$. Mais l'attrait compulsif pour les images ready-made témoigne aussi d'une prise de conscience - contemporaine du développement des Visual Studies - de l'agentivité des images de tous ordres, contribuant à la construction des convictions et des imaginaires collectifs. Les travaux de W. J. T. Mitchell reposent sur l'idée d'un « commun » des représentations visuelles, capables de jeter « un voile idéologique entre le monde et nous ${ }^{2}{ }$ et de jouer un rôle structurant au sein des sociétés. Horst Bredekamp insiste quant à lui sur le fait qu'une fois réalisées, les images gagnent une certaine indépendance pour devenir «agissantes ${ }^{3} »:$ loin de toute hiérarchisation des productions, la culture visuelle nécessite donc une herméneutique, qui enjambe les frontières disciplinaires.

Chez un certain nombre d'artistes contemporains, se situant dans un rapport de filiation plus ou moins direct avec le mouvement conceptuel, le choix de ne pas fabriquer de nouvelles images, mais de collecter, reprendre, réagencer, voire manipuler celles qui existent déjà signe une volonté d'essayer de comprendre les modes de fonctionnement des images, ainsi que ce qu'elles révèlent de la société où elles sont produites. Ce type d'entreprise sonne comme une tentative d'archéologie de la culture où les représentations se donnent comme des documents à ausculter selon des modalités concertées : glanées au marché aux Puces, découpées dans des magazines ou encore téléchargées sur le web, les images sont collectionnées ; elles peuvent dès lors être diversement classées ou agencées selon des dispositifs variés ; parfois elles se trouvent également manipulées; des fonds pour partie fictifs peuvent encore être inventés. Ces 
différentes procédures se présentent comme autant de façons d'essayer de penser les images qui contribuent à façonner les mentalités.

Dans certains cas, les représentations sont réutilisées telles quelles (ou à peine débarrassées des tâches ou des traces d'usure qui les maculent), alors que d'autres fois elles sont transformées de façon substantielle. Mais ces différentes conduites sont prises dans une comparable pulsion de « réactivation » de la puissance évocatoire des images. Si des disparités existent entre les démarches, elles concernent la plus ou moins grande place accordée à des considérations portant sur la plasticité des représentations plutôt qu'à leurs sujets. Les pratiques de remploi ne sauraient être classées selon un gradient d'authenticité (ou de fidélité) aux propositions visuelles initiales. Elles s'attellent en effet toujours à instituer des biais sensibles de compréhension des visions du monde portées par les images. Autrement dit, le recours au trucage, s'il a lieu, ne s'accompagne pas d'une moins grande tension vers la saisie du réel, l'efficacité en ce domaine ne passant pas par un partage entre conformité et falsification, mais par la capacité des œuvres à mobiliser le spectateur vers une intellection de la réalité complexe des phénomènes donnés à voir.

Les documents visuels (récents ou anciens) sont auscultés et infiniment réinterrogés par les artistes. Le remploi compulsif d'images d'origines diverses signe un penchant à la réflexion critique et distanciée sur les artefacts visuels qui contribuent à construire nos mentalités, comme sur les événements photographiés qui font notre histoire. Il témoigne d'une attention portée à des périodes qui se sont écoulées, ou encore à l'état actuel de la société, dans la manière dont il est en train de basculer dans le passé et de s'inscrire dans l'histoire. Ce type de démarche trahit donc une relation au temps, un « régime d'historicité ${ }^{4} »$ qui tend aujourd'hui à prévaloir : les utopies ne sont plus de mise ; le présent, pris dans un mécanisme de péremption de plus en plus rapide, suscite toujours davantage d'incompréhension, l'avenir est envisagé avec inquiétude ; les traces du passé (même le plus proche) semblent dès lors devoir être compulsivement réexaminées afin de prendre appui et de parvenir peut-être à une intelligibilité de la situation actuelle.

\section{Collectionner / Classer}

Dès la fin des années soixante, Gerhard Richter se livre à la collecte systématique d'images sans qualité, de coupures de journaux et de croquis, combinés à des clichés dont il est l'auteur. Les milliers de représentations de toutes catégories, qu'il a peu à peu amassées, ont été régulièrement exposées sous forme de panneaux disposés dans l'espace ou publiés en Atlas ${ }^{5}$ - cette catégorie éditoriale correspondant à des recueils d'images, organisées en planches, dont 
la visée est de permettre un accès au savoir. Si Richter utilise des pièces de cette vaste collection comme sources pour certaines de ses réalisations picturales, le corpus des représentations recueillies constitue également une œuvre à part entière. À la même période, Hans-Peter Feldmann s'adonne au recueil méthodique de photographies d'amateurs, de cartes postales, d'affiches ou de coupures de presse. En collectionneur obsessionnel de l'imagerie populaire, il s'approprie des images trouvées qu'il accumule et agence dans de modestes carnets réalisés à la main - les Bilder - dont chacun regroupe des images, exemptes de légende, représentant un même sujet : genoux de femme, chaussures, chaises ou encore stars de cinéma... À partir des années 80, Joachim Schmid travaille également à partir de vues glanées dans la rue, au marché aux Puces ou encore sur internet qu'il assemble en de petits livres, correspondant souvent à des catégories iconographiques incongrues.

C'est dans la filiation de ces «chiffonniers » des images vernaculaires que s'inscrit « documentation céline duval ». Cette dénomination - adoptée par l'artiste à sa sortie de l'École des Beaux-Arts de Nantes, en 1998 - s'écrit sans majuscule, la jeune femme suggérant ainsi qu'il n'y a pas de hiérarchie entre les lettres, comme il n'y a pas de hiérarchie entre les images. L'appellation constitue en elle-même un programme, puisqu'elle sonne comme la désignation d'une institution dont l'utilité sociale serait de mettre à disposition une archive visuelle. Un tel intitulé entretient la confusion, signant la volonté de la jeune femme de penser son travail artistique comme celui d'un organisme de documentation. La banque d'images progressivement constituée - gigantesque work in progress - s'établit ainsi entre simplicité et démesure, jeu et réalité. La fiction est entretenue d'un certain professionnalisme - qui n'est pas loin d'être devenu véritable, puisque l'artiste a effectivement créé en 1999 une micro-entreprise du nom de «documentation céline duval». Elle estampille d'ailleurs les images qui passent entre ses mains du tampon de l'organisme et son site est structuré de façon efficace et ordonnée, à la manière de celui d'une bibliothèque ${ }^{6}$. Une photographie légendée «L'art du classement, autoportrait par procuration »(2011) la représente, sous une lumière bleutée, en archiviste austère mais sexy, prenant la pose devant un mur de dossiers sévères, rigoureusement alignés.

Les images collectées par Céline Duval sont des photographies de famille, des vues touristiques, des illustrations de magazine ou des cartes postales trouvées de-ci, de-là. Très tôt, à la fin de ses études, la jeune femme se définit comme «iconographe », alors que le terme ne connaît pas la fortune qu'il rencontre aujourd'hui dans le monde de l'art ${ }^{7}$. Si la banque d'images de Céline Duval inclut des photographies de son cru, l'artiste dit en produire de moins en moins puisqu'elle trouve des vues toutes faites qui lui conviennent. Au sein de modestes parutions, 
d'installations voire de diaporamas, les vues se trouvent regroupées en fonction de similarités de sujets.

Céline Duval est attachée à l'imprimé, en raison de son faible coût et de ses potentielles capacités de diffusion. De 2001 à 2009, paraît la Revue en 4 images qui ne compte pas moins de soixante livraisons et assemble des photographies de famille. Chacune de ces humbles parutions est constituée d'une feuille de format A3, pliée en quatre, présentant quatre photographies aux motifs plus ou moins analogues : ébats sportifs sur la plage, jeux de ballon, individus posant à côté d'une automobile, etc. Les abonnés reçoivent les numéros qui leur reviennent par la poste. Depuis 2015, l'ensemble des livraisons peut être acheté sous la forme d'un seul volume relié que l'on peut commander sur le site de l'artiste. En 2001 et 2002, Céline Duval réalise également sept Cahiers d'images avec Hans-Peter Feldmann. Comme les numéros de la Revue en 4 images, ces « petits livres ${ }^{8}$ » présentent des regroupements fondés sur des similitudes iconographiques. À chaque fois, les images reproduites sont minutieusement débarrassées des traces d'usure qui les maculent; elles perdent ainsi un peu de leur existence matérielle pour s'en tenir à une manière de figurer les choses.

Figure 1 : documentation céline duval, double-page de la Revue en 4 images. Avec l'aimable autorisation de l'artiste.

Dès lors que des représentations parentes sont rapprochées les unes des autres, ressortent les mécanismes d'une relative stéréotypie. Se dégagent des catégories d'images ou de scènes au sein desquelles les différences observables ne sont plus que simples variations. L'assemblage de certaines images établit ainsi une hiérarchie des traits figurés : certains se donnent comme constitutifs d'une catégorie, tandis que d'autres semblent relever de l'accident. Le classement, même inchoatif, se présente donc toujours comme une façon de penser les objets organisés. Dans le cas présent, les catégories mises en place reposent toujours sur les éléments représentés (et non par exemple sur les couleurs ou les techniques employées) ; les images se trouvent ainsi appréhendées comme des documents concernant le monde et sur les manières de le voir - qui permettent de penser les scènes représentées dans leur ancrage temporel ainsi que la relation aux images (aussi bien leurs modalités de production que de réception). Dé-singularisant le donné visuel pour l'inscrire dans un type, dégageant l'efficacité des images, les classements de Céline Duval prennent une dimension historique, voire anthropologique.

Pendant de longues années, l'artiste a également collectionné les images publicitaires des magazines féminins. Entre 1998 et 2010, elle travaille à la réalisation d'un ensemble de vidéos en plans fixes - intitulé Les Allumeuses - où l'on voit juste sa main saisir, une à une, les images empilées sur le bord d'une cheminée, puis les froisser avant de les jeter au feu. Le foyer 
lui-même n'est pas filmé : la combustion a lieu hors-champ, mais les flammes voisines jettent leurs reflets orangés sur le papier glacé. Les images publicitaires défilent sous le regard du spectateur, avant d'être brûlées. L'œuvre se présente comme l'enregistrement d'une performance, constituant une sorte de rituel d'anéantissement des images. Mais elle opère, dans le même temps, une « remédiation ${ }^{9}$ » des publicités qui, filmées, sont rendues portées au regard d'un public plus large. Les images kitsch apparaissent dans l'ordre concerté où elles ont été empilées. L'ordonnancement préalable des pages fonctionne ainsi comme une forme de montage, les effets de « raccord » ayant été minutieusement pensés. Chaque vidéo - intitulée « détail ${ }^{10} »-$ correspond à un topos iconographique - yeux cachés, femmes et troncs, ingestion de nourriture, femmes caressant les talons hauts de leurs escarpins, etc. - dont le retour signe la prégnance dans l'imaginaire collectif. Dans la presse féminine, les stéréotypes sont légions et les classements de Céline Duval font ressortir le poids écrasant des poncifs machistes dans ce type de parutions.

\section{Agencer}

Depuis quelques années, l'œuvre d'Aby Warburg a été redécouverte. L'attention s'est portée sur l'organisation de sa bibliothèque - où les ouvrages sont distribués dans l'espace de façon à favoriser la mémorisation des connaissances. C'est aussi L'Atlas Mnémosyne ${ }^{11}$ conçu par Warburg à la fin de sa vie (entre 1924 et 1929) qui suscite la réflexion. Georges DidiHuberman a substantiellement contribué à l'exégèse de cette gigantesque entreprise ${ }^{12}$. Si les agencements d'images proposés aux murs de la salle de conférence de la bibliothèque de Warburg constituaient un outil pédagogique, leur disposition, continuellement remaniée en fonction de la découverte d'affinités jusque-là restées inaperçues, formait également un instrument heuristique, les rapprochements opérés travaillant sans cesse à la genèse d'interprétations renouvelées. Les planches instituaient ainsi un mode de pensée sur les images, par les images. Warburg prévoyait la publication d'un atlas fait de planches non reliées, qui n'a pu voir le jour avant son décès. Pour Didi-Huberman,

Le tableau serait [...] l'inscription d'une œuvre [...] qui se veut définitive au regard de l'histoire. La table [que l'on peut comparer à la planche] n'est que le support d'un travail toujours à reprendre, à modifier si ce n'est à recommencer. Elle n'est qu'une surface de rencontres et de dispositions passagères [...]. L'unicité du tableau fait place, sur une table, à l'ouverture toujours reconduite de nouvelles possibilités, de nouvelles rencontres, de nouvelles multiplicités, de nouvelles configurations ${ }^{13}$. 
Par-delà les chronologies apprises, les échos et les interférences qui se tissent entre les images s'avèrent fécondes pour la réflexion; les planches se font ainsi «machine [s] de lecture ${ }^{14}$ ». Figure 2: Batia Suter, Surface Series (Table Selection), 122 x 244 cm, 2010-2011. Avec l'aimable autorisation de l'artiste.

Dès la fin des années 90, les œuvres de Batia Suter manifestent l'influence de L'Atlas Mnémosyne. L'artiste suisse collectionne les livres illustrés les plus variés dont elle extrait des images afin de constituer une énorme banque de représentations visuelles. L'ampleur de l'archive mobilise un imaginaire borgésien : les représentations ressortissent aux champs de la sculpture, de la botanique, de la géologie, de l'architecture, etc. de sorte que leur accumulation véhicule l'idée d'une forme de maîtrise encyclopédique du monde. Suter redistribue ensuite, à sa guise, les images au sein de livres ${ }^{15}$, de panneaux muraux ou encore de tables placées à quelques centimètres du sol au sein des espaces d'exposition ${ }^{16}$. Ces œuvres suivent la logique du montage, les agencements provisoirement adoptés reposant sur des congruences thématiques ou formelles. Les rapprochements qui se nouent au sein des dispositifs élaborés amènent à réfléchir sur les mécanismes de reconnaissance des motifs visuels comme sur les modalités d'interprétation des images. Les travaux de Suter interrogent la façon dont le cerveau humain traite les informations visuelles.

Figure 3 : Arno Gisinger, vue de 1'exposition « Nouvelles histoires de fantômes », Palais de Tokyo, 2014. Avec l'aimable autorisation de l'artiste.

Le photographe Arno Gisinger a, quant à lui, longuement travaillé avec Georges DidiHuberman, avec L'Atlas Mnémosyne pour source d'inspiration. Une première exposition intitulée «Atlas » a été conçue par le théoricien au Musée Reina Sofía de Madrid ${ }^{17}$ en 2010, en hommage à l'œuvre de Warburg. Didi-Huberman s'est ensuite tourné vers Gisinger, afin de présenter au Studio du Fresnoy une nouvelle version de l'exposition où la photographie tienne une place plus importante - tant il est vrai que l'entreprise warburgienne n'est possible qu'au travers de l'usage de ce médium. Il s'agissait également de montrer combien les dispositifs imaginés par l'historien de l'art sont mobiles et infiniment changeants. À cette fin, Gisinger a photographié l'exposition de Hambourg, au moment de son montage, pendant sa présentation, puis au cours de son démontage. Au Fresnoy, «Atlas, suite » combine en 2012 des images fixes ou mobiles, projetées à même le sol, comme en un gigantesque tapis mouvant, et des tirages photographiques sur dos bleu, collés au mur, présentant les différentes phases de l'exposition de Hambourg.

En 2014, au Palais de Tokyo, Didi-Huberman et Gisinger présentent « Nouvelles histoires de fantômes ». Cette nouvelle exposition s'ouvre sur la projection murale de la planche 
42 de L'Atlas Mnémosyne, consacrée au «pathos de la douleur»: cette mise en exergue annonce qu'il s'agit de s'inspirer du dispositif warburgien pour l'étendre à l'espace muséal. L'aire centrale du Palais de Tokyo est réservée à la projection au sol d'un ensemble d'images filmiques ou picturales, figurant des motifs pathétiques. Le visiteur peut marcher sur cette aire, son ombre venant alors occulter certaines portions des images projetées à terre. Tout autour de cette zone, court une frise de photographies réalisées par Gisinger (certaines d'entre elles montrant le montage et le démontage de l'exposition de Hambourg) : ce dispositif semicirculaire rappelle le modèle panoramique des grandes peintures du XIX ${ }^{\mathrm{e}}$ siècle. Les images réunies dans l'espace d'exposition peuvent ainsi être appréhendées selon une multiplicité de points de vue et des modalités d'implication corporelle variées. Dans le même temps, le dispositif incline le visiteur à une prise de distance devant la variété des images proposées qui l'amène à réfléchir aux mécanismes de retours et de survivances qui se manifestent dans l'histoire des représentations, selon des modalités erratiques et discontinues. L'ordonnancement de l'exposition favorise l'activité aussi bien physique qu'intellectuelle du visiteur.

Des représentations visuelles accumulées peuvent encore être combinées par certains artistes au sein d'œuvres vidéo : Grosse fatigue ${ }^{18}$, réalisée par Camille Henrot en 2013, propose une prolifération d'images appartenant au Smithsonien Institute de Washington (où elle a été en résidence); les représentations défilent et se superposent continûment, accompagnées de la lecture d'un poème en spoken word. Les images qui déferlent, surgissant parfois sous forme de рор-up, sont de sources très variées : registres populaire et savant se croisent, cultures du monde entier voisinent, dans un syncrétisme brouillon. Défilement ou superposition des images à l'écran s'avèrent propices au développement d'associations entre les représentations qui alimentent les imaginaires. Pourtant, davantage qu'à une remontée des filiations ou à une saisie des parentés, Grosse fatigue semble inviter au retrait, sous l'effet de la saturation visuelle.

Mais, la plupart du temps, sous le signe des travaux de Warburg, se développent des œuvres liant intimement modalités d'agencement et nouvelles formes de production de connaissances. Les dispositifs de présentation élaborés permettent de déceler des homologies ou des variations, des échos et des interférences (loin des seules relations causales ou linéaires) : ils conduisent à penser les images à nouveaux frais. Le processus d'investigation paraît alors infiniment ouvert et rend sensible une forme de vie des images qui se développerait sur le mode du réseau pour innerver les mentalités.

\section{Manipuler}


D'autres artistes, fascinés par les images préexistantes, n'hésitent pas à retoucher, transformer les épreuves dont ils héritent afin d'en réactiver la présence et la portée. Une des premières artistes françaises à s'adonner à la « post-photographie ${ }^{19}$ » est Catherine Poncin. Dès les années 80, elle part de vues anonymes collectées au marché aux puces ou dans des albums de famille, de cartes postales ou encore de clichés conservés dans des archives. Pour la série Clair-obscur, mémoire des fosses ${ }^{20}$, ce sont des vues de mineurs (conservées au sein du fonds iconographique du Centre de Lewarde) que Catherine Poncin reprend et manipule. Pour Corps de classe $^{21}$, ce sont des photographies scolaires (rassemblées dans le fonds photographique du Musée dauphinois de Grenoble) qu'elle recadre et ré-agence. Plus tard, la série 1418. Échos,

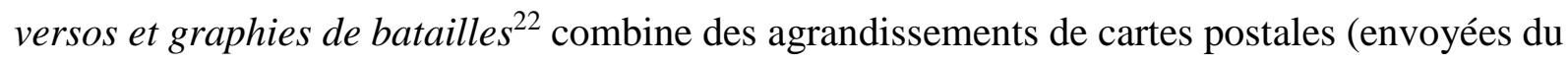
front) à des fragments de correspondance écrite sur leur verso.

Figure 4 : Catherine Poncin, Éloge des combats ordinaires, 2008. Avec l'aimable autorisation de l'artiste.

Les images dont elle s'empare, Catherine Poncin n'hésite pas à les recadrer, afin de faire ressortir certaines coprésences à l'intérieur du champ ou de dilater certains détails. Grossir certaines zones permet de rendre certains éléments visibles - à l'instar de ce qui se passe dans le film Blow up. L'agrandissement intensifie et révèle ce qui était déjà là, tapi dans la représentation, mais qui restait jusqu'alors in-vu. L'image obtenue par le biais d'un enregistrement - qu'il soit argentique ou numérique - procure le sentiment que des faits passés (longtemps ignorés) peuvent, bien après la prise de vue, être ressaisis en son sein afin d'être réouverts à l'attention. Le remploi conjugué au grossissement de certaines zones des photographies semble ainsi pouvoir révéler des données enfouies, comme s'il s'agissait d'une fouille dans l'épaisseur du temps. Une telle démarche paraît porter en elle une approche du passé, aimantée par la singularité de réalités ordinaires et partant des vestiges contenus au sein du présent pour remonter vers des faits anciens, proche de celle que défendait Walter $\operatorname{Benjamin}^{23}$. Le travail de Catherine Poncin touche à «l'étrange alchimie », « qui organise un lien entre le passé et le présent, en dehors de la Mémoire organisée $[\ldots]^{24} »$, décelée par 1'historienne Arlette Farge.

L’artiste Agnès Geoffray se montre fascinée par la force évocatoire des photographies, tout particulièrement celles qui témoignent de relations de domination ou de brutalité. Une

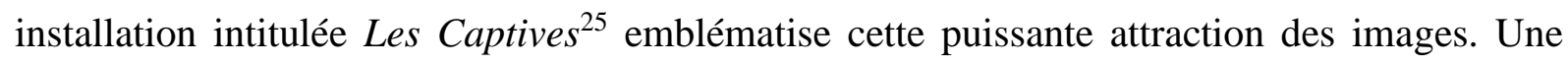
quinzaine de clichés anciens y sont présentés sous cloches de verre soufflé, disséminées dans l'espace. Ces enveloppes translucides semblent protéger les épreuves comme des biens précieux, en même temps qu'elles les mettent hors d'atteinte. Les irrégularités du verre soufflé 
font partiellement écran et obligent les visiteurs à réajuster leur position afin de discerner au mieux les sujets représentés ; ces efforts du regard signent peu ou prou la manière dont les images méritent d'être sondées selon différents points de vue (au propre comme au figuré) pour être perçues. En effet, la question de la distance ou du point de vue, si elle concerne le corps, métaphorise aisément une forme de positionnement intellectuel ou affectif.

Si l'action exercée sur les images reste restreinte dans Les Captives, Agnès Geoffray intervient souvent sur les représentations visuelles de façon plus marquée. C'est par exemple le cas pour Treize fragments. L'installation comprend plusieurs constituants dont une photographie montrant une femme nue conspuée par la foule, à la libération. Chacun a déjà vu des clichés de ce type, témoignant de la façon dont les femmes ayant eu une relation avec un allemand étaient prises comme bouc-émissaire. Mais l'œuvre d'Agnès Geoffray tend précisément à réactiver la violence de la scène photographiée. L'artiste a en effet isolé, au sein de cette épreuve, treize fragments présentés sous forme de diapositives 6 x 6 dans des petites tables lumineuses accrochées au mur. L'agencement de l'installation est tel que le visiteur découvre d'abord les détails rétroéclairés avant de parvenir à la photographie dans son intégrité. Introduite de la sorte, celle-ci retrouve toute sa densité événementielle. Un à un, les détails sont scrutés ; il s'agit d'un index pointé vers la femme nue, d'une expression goguenarde ou d'un poing serré qui n'ont pas nécessairement été retenus de façon consciente par l'opérateur ; dans leur contingence, ils ramènent à la réalité effective de la situation qui a été fixée par l'empreinte photo-chimique - dont l'intensité se trouve ainsi ravivée.

Figure 5 : Agnès Geoffray, Incidental Gestures, 2020. Avec l'aimable autorisation de 1'artiste.

Incidental Gestures est une série constituée d'images anciennes choisies par Agnès Geoffray pour la violence qu'elles recèlent. Mais, ces épreuves, l'artiste les retouche afin de leur donner une étrangeté qui inquiète. C'est ainsi qu'un cliché représentant une femme noire pendue a été manipulée de façon à faire disparaitre la corde qui retient le cadavre. La victime semble dès lors flotter dans les airs de façon quasi surnaturelle. Une autre épreuve qui figure une jeune fille violemment arquée vers l'arrière, dans une posture improbable, résulte de la suppression d'un élément qui lui servait de support. L'attitude du modèle parait dès lors très étrange, faisant penser à celles des cataleptiques ou des hystériques dans les vues médicales réalisées au dix-neuvième siècle. Les manipulations pratiquées surprennent et réquisitionnent l'attention du spectateur, happé par l'étrangeté des images - renvoyant à une singularité du photographié qui refuse d'être rangé dans une catégorie générale et réclame une existence passée effective. Les modifications opérées par Agnès Geoffray recréent une tension dramatique, réactivent le poids de réalité des représentations. 
C'est également à une régénération des images que se livre Pavel Maria Smejkal ${ }^{26}$. Cet artiste reprend des vues emblématiques, des icônes de l'histoire, trouvées sur internet, dont il efface le motif principal. C'est ainsi que la photographie d'un soldat républicain réalisée par Capa en 1938 se voit privée de la présence de son personnage principal, de sorte que ne reste que la pente rase d'une colline. De même le cliché réalisé par Eddy Adams à Saïgon en 1968 représentant un vietminh au moment même où il est mis à mort se trouve nettoyé de ses acteurs. Mais ces images ont tellement été vues que le spectateur, qui fouille dans sa mémoire, réussit à les convoquer mentalement, ou en tout cas ressent le manque des éléments qui sont désormais absents. Paradoxalement, l'effacement redonne une force à l'image face à laquelle l'anesthésie était devenue la règle. Le truquage qui gomme certains éléments essentiels rend l'existence passée des faits plus vivante dans les esprits. C'est comme si l'on avait imaginé un instant que, selon les modalités d'une histoire alternative, ces événements auraient pu ne pas advenir... Leur horreur frappe ensuite avec d'autant plus de force.

\section{Fabuler}

Dans le cadre des Rencontres Internationales de la Photographie 2014, Le Musée Départemental Arles Antique propose un aperçu de la «Collection Trepat», sous le commissariat d'un dénommé Joan Fontcuberta ${ }^{27}$. Sous-titrée «Un cas d'étude de la photographie d'avant-garde », l'exposition est censée livrer les pièces essentielles du fonds constitué au début du vingtième siècle par l'industriel catalan Josep Trepat, comprenant des vues de son usine de machines agricoles, des brochures d'entreprise, des documents publicitaires, mais également des œuvres commandées aux plus grands artistes de l'avantgarde. Cet ensemble traduit la proximité des usages de la photographie au service de l'industrie et des travaux, circulant alors dans le monde de l'art, tournés vers une apologie de la machine et du progrès.

Mais cette exposition tient de la « chimère » puisqu'aux documents visuels trouvés dans une véritable entreprise ${ }^{28}$, Foncuberta mêle des forgeries, des vues qu'il a manipulées voire fabriquées. Certaines images se présentent même comme le pastiche d'œuvres connues. C'est par exemple le cas d'un cliché montrant une jeune femme nue, accoudée à une machine, dont l'avant-bras et la main sont maculés de noir - qui renvoie au fameux portrait de Meret Oppenheim intitulé «Érotique voilée », réalisé par Man Ray en 1933. Certains collages reprennent de façon ludique les portraits détourés d'acteurs connus de 1'avant-garde (Albert Renger-Patzsch, László Moholy-Nagy, Charles Sheeler...) pour les placer devant une vue des établissements Trepat - dont on pense dès lors qu'ils les ont visités. 
Mais par-delà telle invention, tel montage ou trucage particuliers, c'est tout le dispositif scénographique qui se trouve organisé afin de créer la fiction d'un fonds d'images de l'industrie, agrémenté de belles réalisations commandées à des artistes - les différentes pièces de l'exposition faisant sens les unes par rapport aux autres. Pour John Searle, au sein d'une fiction, le principe de cohérence interne l'emporte sur les relations « vérifonctionnelles » - où il s'agit de soupeser l'authenticité des différents éléments combinés. Autrement dit, les rapports de type « horizontal » tendent à occulter les connexions «verticales » qui lient le langage à la réalité (ces dernières prédominant dans d'autres types d'actes illocutoires) ${ }^{29}$. Pourtant la fiction n'exclut pas la référence au réel, puisque c'est elle qui permet que l'univers diégétique soit envisagé comme une forme de prolongement du monde. À ce fonds dont un échantillon lui est proposé, le visiteur est invité à imaginairement donner corps : il «fait comme si », s'adonnant à une forme de «feintise ludique ». L'exposition se donne comme un espace de jeu.

Ce n'est pourtant pas si simple car le fonds proposé s'avoue vite comme un «leurre », plus précisément comme un « leurre qui échoue à duper complètement le visiteur ». Jean-Marie Schaeffer distingue «feintise ludique » et «feintise sérieuse ${ }^{30} »$ : la première - correspondant à la fiction - suppose une forme de consensus entre l'auteur et le spectateur qui s'accordent sur un «faire comme si »; la seconde, la «feintise sérieuse » prétend faire passer le faux pour le vrai ; elle est tromperie, leurre. La présentation de la «Collection Trepat», tout en donnant à imaginer un fonds fictif, fonctionne peu ou prou comme une tentative de supercherie, face à laquelle le visiteur serait amené à exercer ses facultés de discernement. L'exposition étant constituée d'éléments discrets, combinés les uns aux autres, l'authenticité de chacun d'entre eux se trouve plus ou moins soupesée par le visiteur - qui essaie d'évaluer s'il s'agit de documents d'époque ou de contrefaçons. En proie au doute, le spectateur se mue en détective.

Si l'expérience de la visite prend cette tournure, c'est que le contexte de l'exposition fonctionne comme sommation à prendre les choses au sérieux. Le Musée Départemental Arles Antique est un établissement à vocation historique. Le cadre dans lequel s'insère le dispositif de monstration a l'allure d'un piège qui accule presque le visiteur à adhérer à la version qui lui est proposée. De surcroît, l'exposition d'archives de l'histoire de la photographie constitue aujourd'hui un genre, auquel la monstration de la « Collection Trepat» semble appartenir. La fiction suppose en général l'introduction d'éléments référentiels dans un univers fictionnel afin de lui donner corps. Ici, c'est l'inverse : des éléments fabriqués ou manipulés sont insérés dans un cadre dont il est globalement posé qu'il a « valeur documentaire ». Les forgeries apparaissent donc comme des dérogations à une authenticité qui est de mise. Le médium photographique travaille en outre à convaincre de la véracité du représenté. Dès lors, les manipulations repérées 
semblent relever d'une opération de falsification ratée, d'un bricolage imparfait. Leur identification par le visiteur enclenche le renvoi à une poḯtique de l'œuvre - que constitue l'exposition - où le commissaire Fontcuberta se présente en faussaire. La réputation de l'artiste catalan ne peut évidemment que peser en ce sens.

Ce sont les tromperies les plus flagrantes qui mettent «la puce à l'oreille». Les improbables portraits de Walker Evans ou d'Alexander Rodchenko prenant la pose devant les ateliers mécaniques, le renvoi parodique à des œuvres connues retiennent l'attention et travaillent à saper la cohésion de l'ensemble; ces photographies ne peuvent se fondre à l'archive documentaire; mais ils résistent aussi à s'intégrer à un univers inventé, puisqu'ils ressortissent de manière flagrante à l'histoire de l'art. Certains anachronismes tendent encore à interroger le visiteur. Dès lors les forgeries ne se présentent pas comme des «indices de fictionnalité ${ }^{31} »$, mais davantage comme des «symptômes de tromperie » qui rétroagissent sur la perception de l'ensemble.

L'exposition n'est pas la présentation d'un fonds industriel fictif, ni même la fiction d'une archive particulièrement riche ; il s'agit plutôt de la fiction d'une « supercherie ratée ». Si Fontcuberta avait voulu tromper ses visiteurs en enrichissant sensiblement l'archive, il aurait pu y parvenir aisément. Mais il a pour ainsi dire choisi de se mettre en scène, de façon ludique, en commissaire-faussaire. L'orchestration d'un (relatif) échec du leurre ne gomme cependant pas la charge didactique de l'exposition : l'évocation du fonds d'un industriel proche des travaux d'artistes de l'avant-garde demeure. Mais le choix d'un savant emboîtement des niveaux n'est pas sans conséquences puisqu'il incline le visiteur à une oscillation entre adhésion à l'histoire inventée, suspension de l'incrédulité et repérage de la supercherie : l'ambiguïté travaille à la prise de distance comme à la fragilisation de la frontière séparant ordinairement le monde de la fiction de celui de l'histoire. Certaines pièces montrées sont des images d'archive, d'autres vues sont inventées, mais elles le sont à partir de matériaux relevant de l'histoire de l'art. La réalité irrigue donc de différentes façons la fiction et, dans une certaine mesure, la circonspection engendrée par la forme du leurre déclenche une attitude d'enquête qui fait également du passé son objet.

Si bien des artistes contemporains témoignent d'un « mal d'archive ${ }^{32} »$, ils n'empruntent pas les mêmes chemins quand ils manient, et remanient, les documents visuels dont ils s'emparent : nombreux sont ceux qui collectent, voire collectionnent ; certains classent, d'autres agencent en dispositifs concertés ; il en est qui manipulent et retouchent les images déjà-là, 
tandis que d'autres fabulent et forgent de vrais « faux fonds ». Toutes ces opérations relèvent d'une praxis de l'archive : si elles peuvent être caractérisées, elles ne sauraient être distinguées selon un gradient d'authenticité. Le fait de rassembler certaines vues figurant un même motif (Céline Duval) se présente déjà comme un geste d'interprétation des images qui ne les laissent pas indemnes. La disposition des images constitue une autre forme d'invention (Batia Suter, Arno Gisinger). Le recours à tel ou tel trucage ne trahit pas une moins grande soif de justesse (Agnès Geoffray), puisque la capacité à faire retour sur le réel ${ }^{33}$ découle de l'aptitude des œuvres à mobiliser le spectateur vers une compréhension de la complexité des phénomènes, tels que les images les font advenir au regard. Les travaux de Céline Duval, Batia Suter, Arno Gisinger, Catherine Poncin, Agnès Geoffray ou Joan Foncuberta... travaillent tous à réactiver l'agentivité des images, à exalter et comprendre l'importance qu'elles revêtent au sein des modes de pensée.

Quelle que soit la manière dont il opère, le remploi compulsif signe un effort de réflexion sur les artefacts visuels qui contribuent à régir les mentalités. Une fois détachées de leur situation de production, les images tendent à s'émanciper et à circuler au sein de la société : elles hantent les livres, les réseaux et les esprits, leur portée évoluant selon les contextes. C'est la vitalité quasi organique des représentations visuelles que les œuvres d'art ici prises en considération auscultent et réactivent. Selon une démarche proche de l'anthropologie, les usages des représentations se trouvent questionnés - la recherche sur les images progressant par le biais du recours aux images. La manière dont événements historiques ou faits sociaux s'incarnent dans certaines vues est également questionnée, les images se présentant comme des relais pour penser les faits et la manière dont ils sont consignés dans les mémoires.

Les représentations visuelles reprises par ces artistes sont le plus souvent des photographies. Le caractère indiciel des images ainsi réactivées se met au service de leur inscription dans le temps. Spectrale, l'épreuve photographique renvoie par métonymie au moment révolu de la prise de vue comme aux sujets qui ont été fixés. L'observation minutieuse et détaillée du cliché d'une femme nue, conspuée par la foule, au moment de la libération amène à ressentir la singularité de l'événement dans sa violence même, au détriment d'une appréhension plus froide et chronologique des événements. Même quand les scènes figurées sont récentes, elles paraissent en train de basculer dans le passé, de verser d'emblée dans une sorte de patrimoine visuel, protéiforme mais largement opérant, dont l'agentivité mérite d'être auscultée.

Si le médium photographique contribue, par sa nature même, à impliquer l'idée du révolu, la propension actuelle à des formes de patrimonialisation de plus en plus rapides ne 
s'avère pas étrangère aux démarches qui ont été examinées ici. Comme l'a montré Harmut Rosa $^{34}$, les rythmes de la vie sociale se sont accélérés, de sorte que les objets et les comportements sont pris dans des mécanismes d'obsolescence précipités. Ainsi les images d'hier se donnent-elles rapidement comme des témoignages susceptibles d'être réexaminés. Tout étant menacé de péremption rapide, les représentations visuelles prennent, sitôt réalisées, valeur de documents sur des réalités en passe de disparaître. Rares sont aujourd'hui les secteurs qui ne se trouvent pas concernés par l'essor galopant de la patrimonialisation et la tendance à la constitution de «lieux de mémoire ». À cette pente, les images n'échappent pas : comme d'autres artefacts, elles semblent pouvoir être avalées dans une temporalité accélérée, aussi bien dans leur matérialité physique qu'en ce qui concerne leurs sujets.

Cette manière d'envisager les objets de toutes natures, même les plus fraîchement fabriqués, n'est pas sans révéler une inquiétude montante. L'avenir paraît aujourd'hui incertain : de nombreux «lanceurs d'alerte » dénoncent les risques encourus par les sociétés contemporaines ; les adeptes de la « collapsologie » sont de plus en plus nombreux ; les fictions d'apocalypse prolifèrent ${ }^{35}$. Les « grands récits », les « utopies » semblent relever d'une période révolue, et nos concitoyens peinent à se projeter dans le futur ${ }^{36}$. Le monde contemporain paraît caractérisé par la vulnérabilité. Dès lors, les représentations passées ou récentes semblent devoir être compulsivement sondées afin d'y déceler les témoignages d'une évolution qui permettent de comprendre mieux la période actuelle, de lui trouver peut-être une assise.

1 Anne Immelé, "Archives et constellations ", Focales $n^{\circ} 2$ (2018): http://focales.univ-st-etienne.fr /index.php?id=2056

${ }^{2}$ W. J. T. Mitchell, "Montrer le voir. Une critique de la culture visuelle », Que veulent les images ? Une critique de la culture visuelle (Dijon : Les presses du réel, 2014), 346.

${ }^{3}$ Horst Bredekamp, Théorie de l'acte d'image (Paris : La découverte, 2015), 15.

${ }^{4}$ François Hartog. Régimes d'historicité. Présentisme et expérience du temps (Paris : Seuil, 2003).

${ }^{5}$ Gerhard Richter, Atlas (Cologne : Verlag der Buchhandlung Walther König, 2006).

${ }^{6}$ Voir : https://www.doc-cd.net/

${ }^{7}$ Garance Chabert, Aurélien Mole, Les Artistes iconographes (Dijon : Les Presses du réel, 2018).

${ }^{8}$ Anne Mœglin-Delcroix, " "Petits livres" et autres petites publications », Sur le livre d'artiste. Articles et écrits de circonstance (1981-2005) (Marseille : Le Mot et le reste, 2006), 246-285.

9 Jérôme Dupeyrat, "Emprunts et remédiation »: https://www.canal-u.tv/video/universite_toulouse_ii_ le_mirail/emprunts_et_remediation_jerome_dupeyrat.17827 
${ }^{10}$ Voir : http://www.lesallumeuses.net/

${ }^{11}$ Aby Warburg, L'Atlas mnémosyne : avec un essai de Roland Recht (Paris : L'écarquillé-INHA, 2012).

${ }^{12}$ Georges Didi-Huberman, L'Image survivante. Histoire de l'art et temps des fantômes selon Aby Warburg (Paris : Minuit, 2002) ou Atlas ou le gai savoir inquiet (Paris : Minuit, 2011).

${ }^{13}$ Georges Didi-Huberman, Atlas ou le gai savoir inquiet, op. cit., p. 18.

14 Ibid., p. 15.

${ }^{15}$ Batia Suter, Parallel Encyclopédia (Amsterdam : Roma Publication, 2007 et 2016).

${ }^{16}$ Voir : https://www.batiasuter.org/

${ }^{17}$ Cette exposition a circulé au ZKM de Karlsruhe et aux Deichtorhallen - Sammlung Folckenberg de Hambourg.

${ }^{18}$ La vidéo de 13' est en couleur et sonore.

${ }^{19}$ Paul Ardenne, « Catherine Poncin post-photographe », Catherine Poncin (Trézélan: Filigranes, 1999), 4-9.

${ }^{20}$ Série réalisée en 1998-1999 : Ibid., 54-63

${ }^{21}$ Série réalisée en 1999: Ibid., 64-77.

${ }^{22}$ Catherine Poncin, 1418. Échos, versos et graphies de batailles (Trézélan : Filigranes, 2001).

${ }^{23}$ Walter Benjamin, « Sur le concept d'histoire » (1940), Écrits français (Paris : Gallimard, 1991), 343.

${ }^{24}$ Arlette Farge, La Chambre à deux lits et le cordonnier de Tel-Aviv (Paris : Seuil, 2000), 117.

${ }^{25}$ Les séries Les Captives, Treize fragments et Incidental Gestures ont été présentées au FRAC Auvergne en 2020. Voir le catalogue : Agnès Geoffray (Clermont-Ferrand : Frac Auvergne, 2020).

${ }^{26}$ Voir : https://www.lensculture.com/pavel-maria-smejkal-2

27 Parallèlement paraît Trepat (Paris : Bessard, 2014).

${ }^{28}$ L'entreprise Trepat est fondée dans la province de Lérida en 1914 et ferme en 1985. C'est en 2014 (pour la célébration de sa naissance) que la municipalité sollicite Fontcuberta.

${ }^{29}$ John Searle, "Le statut logique du discours de la fiction ", Sens et expression. Études et théorie des actes de langage (Paris : Minuit, 1982), 101-119.

30 Jean-Marie Schaeffer, Pourquoi la fiction ? (Paris : Seuil, 1999).

${ }^{31}$ Käte Hamburger, Logique des genres littéraires (Paris : Seuil, 1986).

32 Jacques Derrida, Mal d'archive (Paris : Galilée, 1995), 142 : être en « mal d'archive », " c'est se porter vers elle d'un désir compulsif, répétitif et nostalgique, un désir irrépressible de retour à l'origine [...] ॥.

${ }^{33} \mathrm{Hal}$ Foster, Le Retour du réel. Situation actuelle de l'avant-garde (Bruxelles : La Lettre volée, 2005).

${ }^{34}$ Harmut Rosa, Accélération. Une critique sociale du temps (Paris : La découverte, 2013).

35 Jean-Paul Engélibert, Fabuler la fin du monde. La puissance critique des fictions d'apocalypse (Paris: La découverte, 2019).

${ }^{36}$ Camille de Toledo, Aliocha Imhoff, Kantuta Quirós, Les Potentiels du temps. Art \& politique (Paris : Manuella, 2016). 